\title{
Discovery of the X-ray burster SAX J1752.3-3138
}

\author{
M. Cocchi ${ }^{1}$, A. Bazzano ${ }^{1}$, L. Natalucci ${ }^{1}$, P. Ubertini $^{1}$, J. Heise ${ }^{2}$, E. Kuulkers ${ }^{2,3}$, \\ R. Cornelisse ${ }^{2,3}$, and J. J. M. in 't Zand ${ }^{2,3}$ \\ 1 Istituto di Astrofisica Spaziale $(I A S / C N R)$, via Fosso del Cavaliere 100, 00133 Roma, Italy \\ 2 Space Research Organization Netherlands (SRON), Sorbonnelaan 2, 3584 CA Utrecht, The Netherlands \\ 3 Astronomical Institute, Utrecht University, PO Box 80000, 3508 TA Utrecht, The Netherlands
}

Received 19 July 2001 / Accepted 28 August 2001

\begin{abstract}
During a 50 ks monitoring observation of the Galactic bulge performed in September 1999 by the Wide Field Cameras on board the BeppoSAX satellite, an X-ray burst was detected from a sky position $\sim 3^{\circ}$ off the Galactic centre. No previously known X-ray sources are located within the position error circle of the observed burst. The new burster, SAX J1752.3-3138, did not show any persistent emission during the whole observation. No other bursting events, as well as steady emission, were reported so far by other instruments or detected in the WFC archive, which covers $\sim 6 \mathrm{Ms}$ and $\sim 4 \mathrm{Ms}$ for burst and persistent luminosity detection, respectively, starting from August 1996. Unless the source is a very weak transient, this could indicate SAX J1752.3-3138 is an atypical burster, a member of a possibly new class of sources characterised by very low steady luminosities and accretion rates $\left(L_{\mathrm{X}} \lesssim 10^{35} \mathrm{erg} \mathrm{s}^{-1}\right)$ and extremely rare bursting activity. The characteristics of the detected burst are consistent with a type I event, identifying the source as a weakly magnetised neutron star in a low-mass X-ray binary system. Evidence for photospheric radius expansion due to Eddington-limited burst luminosity allows to estimate the distance to the source $(\sim 9 \mathrm{kpc})$.
\end{abstract}

Key words. binaries: close - stars: neutron, individual (SAX J1752.3-3138) - X-rays: bursts

\section{Introduction: The BeppoSAX-WFC Galactic bulge monitoring program}

One of the main scientific goals of the Wide Field Cameras (WFC, Jager et al. 1997) on board the BeppoSAX satellite (Boella et al. 1997) is the study of the timing and spectral behaviour of both transient and persistent sources in the Galactic bulge, X-ray binaries in particular, on time scales ranging from seconds to years. To this end, a monitoring program of systematic wide field observation of the Sgr A sky region is carried out since August 1996 (Heise 1998; Heise et al. 1999; Ubertini et al. 1999). The program consists of a series of observations, each lasting 60 ks net time, almost weekly spaced throughout the two visibility periods (August-October and FebruaryApril) of the Galactic Centre region. Up to January 2001, an amount of 4.0 Ms of data has been accumulated this way. The WFC program on the Galactic bulge is significantly contributing in the study of transient X-ray binary systems, mainly X-ray bursters (see e.g. in 't Zand et al. 2000 for a recent overview).

SAX J1752.3-3138 was discovered by the WFCs during the Summer-Fall 1999 monitoring campaign of the

Send offprint requests to: M. Cocchi,

e-mail: wood@ias.rm.cnr.it
Galactic bulge. The source was observed during an X-ray burst (see Figs. 1 and 2, top panel) and no persistent emission was found (Cocchi et al. 1999a). No other X-ray bursts are detected in the whole WFC data archive. The net exposure time available for burst detection is $6.3 \mathrm{Ms}$ up to January 2001, being the attitude constraints not so stringent as for steady emission detection. This makes the observed event the only source of information about SAX J1752.3-3138 available so far. Nevertheless, burst detection itself provides important details on a newly discovered source, being such events uniquely associated to weakly magnetised neutron stars (NS) in Low-Mass X-ray Binary systems (LMXBs). Besides the nature of the source, in several cases X-ray bursts allow to estimate useful parameters such as the source distance and luminosity, and the NS radius (see Lewin et al. 1993, hereafter LvPT93, for a comprehensive review). A single detected burst in a fairly big amount of observing time is even more interesting, since its uniqueness puts important constraints on the characteristics of the accretion onto the neutron star. In the case of SAX J1752.3-3138 in particular, since no steady emission was observed, we explore the bursting behaviour of sources at very low persistent luminosities. The study of the lower luminosity boundary when NS start bursting is 
Table 1. Summary of the main parameters of the observed burst.

\begin{tabular}{lc}
\hline \hline Burst date & 1999, September 2 \\
Burst UT time $(\mathrm{h})$ & 3.3936 \\
$e$-folding time $(2-19 \mathrm{keV})$ & $21.9 \pm 1.3 \mathrm{~s}$ \\
$e$-folding time $(2-6 \mathrm{keV})$ & $21.6 \pm 2.8 \mathrm{~s}$ \\
$e$-folding time $(6-19 \mathrm{keV})$ & $16.0 \pm 2.2 \mathrm{~s}$ \\
peak intensity $(2-19 \mathrm{keV})$ & $710 \pm 32 \mathrm{mCrab}$ \\
blackbody $k T$ & $1.64_{-0.09}^{+0.10} \mathrm{keV}$ \\
$R_{\mathrm{km}} / d_{10 \mathrm{kpc}}$ & $9.2_{-0.9}^{+1.5}$ \\
Reduced $\chi^{2(a)}$ & 1.00 \\
steady emission $^{(b)}$ & $<6 \mathrm{mCrab}^{(c)}$ \\
\hline
\end{tabular}

(a) 26 d.o.f.; (b) MJD 51452.05-51453.21, $3 \sigma$ upper limit, $2-28 \mathrm{keV}$; (c) In the $2-28 \mathrm{keV}$ band, $1 \mathrm{mCrab}=3.17 \times$ $10^{-8} \mathrm{erg} \mathrm{cm}^{-2} \mathrm{~s}^{-1}$.

an important ingredient for our knowledge of thermonuclear bursts.

In the next section we report on the observation and the spectral/timing data analysis of the (so far) only burst of SAX J1752.3-3138. We then briefly discuss the results in Sect. 3, proposing SAX J1752.3-3138 either as a weak transient NS-LMXB or, more intriguingly, as a member of a possibly new class of low-luminosity, slowly accreting $\mathrm{X}$-ray bursters. An estimate of the distance to the source will be also given.

\section{SAX J1752.3-3138: Observation and data analysis}

The BeppoSAX-WFCs are two identical coded aperture multi-wire proportional counters, each covering a $40^{\circ} \times 40^{\circ}$ field of view, the largest ever flown for an arcminute ( $1^{\prime}-3^{\prime}, 99 \%$ confidence) location accuracy X-ray telescope. The cameras point at opposite directions and operate in the $2-28 \mathrm{keV}$ bandpass. The time resolution of the imaging operative mode is $0.488 \mathrm{~ms}$, and the energy resolution is $18 \%$ at $6 \mathrm{keV}$. The imaging capability and the good instrument sensitivity (5-10 mCrab in $10^{4} \mathrm{~s}$ ) allow an accurate monitoring of complex sky regions, like the Galactic bulge.

Thanks to their scientific capabilities, the BeppoSAXWFCs are well designed to systematically investigate transient phenomena lasting from seconds to minutes, like the $\mathrm{X}$-ray bursts. The data of the two cameras is searched for bursts and flares by analysing the time profiles of the detectors with a time resolution down to $1 \mathrm{~s}$. Reconstructed sky images are generated for any statistically significant event, in order to identify possible new bursters. The accuracy of the reconstructed position, which of course depends on the burst intensity, is typically better than $5^{\prime}$. This analysis procedure demonstrated its effectiveness throughout the Galactic bulge WFC monitoring program, leading to the identification of more than $2.2 \times 10^{3} \mathrm{X}$-ray bursts (or $\sim 1.6 \times 10^{3}$ when excluding the Rapid Burster

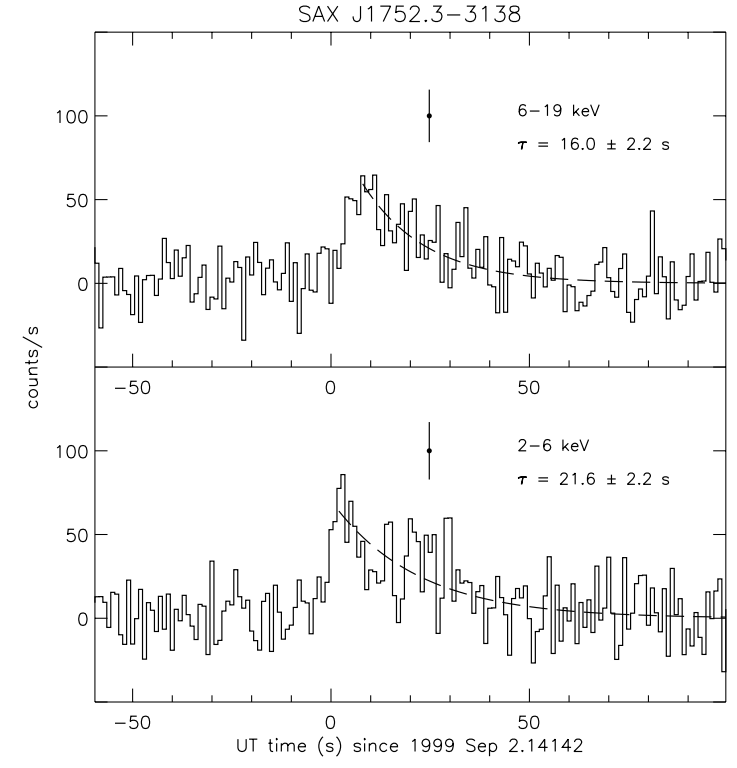

Fig. 1. Energy resolved time histories of the burst. The dashed curves are exponential decay fits to the profiles. The typical error bars are also shown.

and the Bursting Pulsar) from 38 different sources (e.g. in 't Zand et al. 2000).

The X-ray burst from the new source SAX J1752.3-3138 was found during a post-facto analysis of a $50 \mathrm{ks}$ observation of the Galactic centre region performed by the WFC unit 2 on September 2, 1999 (Cocchi et al. 1999a). The bursting source is located $\sim 3^{\circ} \mathrm{SW}$ of Sgr A, at $\alpha=17^{\mathrm{h}} 52^{\mathrm{m}} 24^{\mathrm{s}}, \delta=-31^{\circ} 37.7^{\prime}$ (J2000, error radius $2.9^{\prime}, 99 \%$ confidence). The burst peak intensity, corrected for dead time effects, was $\sim 0.7$ Crab. No steady emission was detected during the whole observation, with a $3 \sigma$ upper limit of $6 \mathrm{mCrab}$ in the $2-28 \mathrm{keV}$ band. No other X-ray bursts were reported from the same sky position during the observation, even if other events could be missed due to the $\sim 53 \%$ source covering efficiency associated to the low Earth orbit of BeppoSAX. Search for bursts and/or persistent emission from SAX J1752.3-3138 was performed on all the data available from the 1996-1999 BeppoSAX-WFC Galactic Bulge monitoring campaigns but, as mentioned above, we report no other significant detections. The typical WFC sensitivity (on-axis) to burst events is approximately $180 \mathrm{mCrab}(2-$ $28 \mathrm{keV})$, corresponding to a peak of $\sim 7 \times 10^{37} \mathrm{erg} \mathrm{s}^{-1}$ ( $\left.\sim 30 \% L_{\mathrm{Edd}}\right)$ for a $10 \mathrm{kpc}$ distance. This value is given for a $6 \sigma$ detection of a burst with exponential decay and characteristic time of $10 \mathrm{~s}$.

In Fig. 1 the time profiles of the burst, obtained in two energy bands, are shown. The time histories of the bursts are constructed by accumulating only the detector counts associated with the shadowgram of the analysed source, thus improving the signal-to-noise ratio of the profile. The background, which is the sum of (part of) the diffuse X-ray background, the particles background and the contamination of other sources in the field of view, has been subtracted. Source contamination is the 


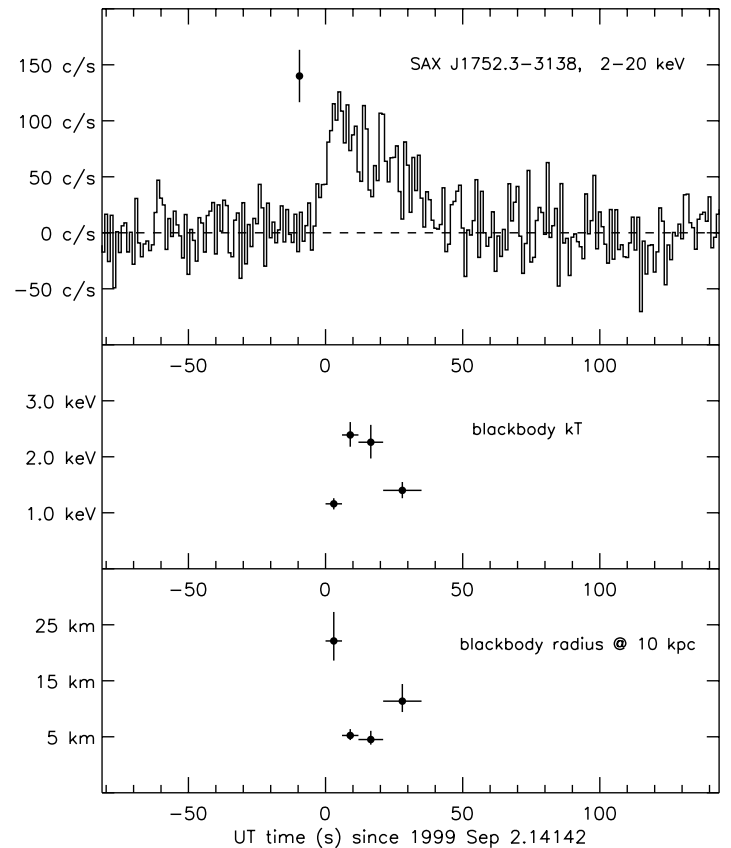

Fig. 2. Time history of the burst, the blackbody colour temperature and the blackbody radius, from upper to lower panel, respectively.

dominating background component for crowded sky fields like the Galactic bulge. Nevertheless, the probability of source confusion during a short time-scale event (10-100 s) like an X-ray burst is negligible.

The burst spectrum of SAX J1752.3-3138 is consistent with absorbed blackbody radiation with average colour temperature of $\sim 1.6 \mathrm{keV}$ (see Table 1 ). Due to the rather poor statistics, the $N_{\mathrm{H}}$ parameter could not be satisfactorily constrained, so it was kept fixed according to the interpolated value for the source sky direction, $5.64 \times 10^{21} \mathrm{~cm}^{-2}$ (Dickey \& Lockman 1990). An absorbed power-law model does not fit the data adequately.

Time-resolved spectra were accumulated for the observed burst, in order to study the time evolution of the spectral parameters (Table 2). The spectra are all consistent with blackbody radiation, and spectral softening is observed during the burst decay, in agreement with the longer decay time associated to the low-energy time profile (see Fig. 1). Blackbody spectra allow to determine the relationship between the average radius of the emitting sphere $R_{\mathrm{km}}$ (in units of $\mathrm{km}$ ) and the source distance $d_{10 \mathrm{kpc}}$ (in units of $10 \mathrm{kpc}$ ). In Fig. 2 the time histories of the measured $R_{\mathrm{km}} / d_{10}$ kpc ratios are shown, assuming isotropic emission and not correcting for gravitational redshift and conversion to true blackbody temperature from colour temperature (see LvPT93 for details).

\section{Discussion}

\subsection{The neutron star $L M X B$ SAX J1752.3-3138}

As anticipated, the spectral and timing properties of the burst detected from SAX J1752.3-3138 are clearly suggestive of a type I event (Hoffman et al. 1978), typically
Table 2. Time resolved spectral analysis of the burst.

\begin{tabular}{lccc}
\hline \hline time range $^{(a)}$ & $k T(\mathrm{keV})$ & $R_{\mathrm{km}} / d_{10 \mathrm{kpc}}$ & $\chi_{\mathrm{r}}^{2(b)}$ \\
\hline $0-6 \mathrm{~s}$ & $1.16 \pm 0.10$ & $22.1_{-3.5}^{+5.2}$ & 0.73 \\
$6-12 \mathrm{~s}$ & $2.39_{-0.21}^{+0.23}$ & $5.2_{-0.8}^{+1.1}$ & 0.65 \\
$12-21 \mathrm{~s}$ & $2.26_{-0.29}^{+0.31}$ & $4.5_{-0.9}^{+1.6}$ & 1.10 \\
$21-34 \mathrm{~s}$ & $1.40_{-0.14}^{+0.15}$ & $11.4_{-1.9}^{+3.1}$ & 0.71 \\
\hline
\end{tabular}

(a) Time since burst start (see Table 1); ${ }^{(b)} 27$ d.o.f.

associated to weakly magnetised neutron stars in lowmass binary systems (e.g. LvPT93). In fact the blackbody emission and the measured colour temperatures of $\sim 1.6 \mathrm{keV}$ are consistent with this hypothesis. Spectral softening is observed in the time resolved spectra of the bursts (Table 2), and the bursts time profiles can be fitted with exponential decays whose characteristic times are energy dependent, being shorter at higher energies (Fig. 1).

The time history of the blackbody radius as obtained from the time resolved spectral analysis of the burst (Fig. 2, lower panel) shows evidence for radius expansion during the very first seconds of the event. This is generally interpreted as adiabatic expansion of the neutron star photosphere during a high luminosity (Eddington-limited) type I burst. The WFC lightcurve of this event is not sensitive enough to show evident flat-top or double-peaked profile (e.g. LvPT93), due the prompt photospheric expansion (see later). An expansion by a factor $\gtrsim 3$ with respect to the average decay radius is observed, while lower luminosity bursts show almost constant blackbody radius during the whole event. The observed expansion is consistent with the time profiles in Fig. 1, as the soft emission precedes by $\sim 4 \mathrm{~s}$ the harder one. Relatively high luminosity and no hard emission $(k T$ is $\sim 1 \mathrm{keV})$ in the very first seconds of the burst imply high values of the blackbody radius. If we narrow the time bin, concentrating on the first $4 \mathrm{~s}$ of the burst, we obtain an even larger radius, $33_{-6}^{+10} \mathrm{~km}$ for a $10 \mathrm{kpc}$ distance. This points to a prompt expansion of the neutron star photospere after the ignition. The fast rise and the prompt photospheric expansion are suggestive of helium burning, while the duration of the burst ( $e$-folding time of $\sim 20 \mathrm{~s}$ ), rather long for a pure helium flash, indicates that the burning environment was not hydrogen-free (LvPT93, Bildsten 2000).

Eddington-luminosity X-ray bursts can lead to an estimate of the source distance, if we assume isotropic emission and an Eddington bolometric luminosity of $2.5 \times 10^{38} \mathrm{erg} \mathrm{s}^{-1}$, which is appropriate for a $1.4 M_{\odot}$ neutron star and helium-rich fuel. This value also includes a moderate (20\%) gravitational redshift correction (van Paradijs \& Mc Clintock 1994). Taking into account the observed peak flux of the burst (Table 1), which extrapolates to an unabsorbed bolometric intensity of $(2.48 \pm 0.21) \times 10^{-8} \mathrm{erg} \mathrm{cm}^{-2} \mathrm{~s}^{-1}$, we obtain $d=$ $9.2 \pm 0.4 \mathrm{kpc}$. The reported error is $1 \sigma$, but the systematic uncertainties due the assumption of standard burst parameters are of course larger. 
Assuming a Crab-like spectrum, we derive for the source persistent (bolometric) luminosity an upper limit of $\sim 4.6 \times 10^{36} \mathrm{erg} \mathrm{s}^{-1}$ when the burst was observed (Sep. 2, 1999). An average upper limit twice as low applies to the whole 1996-2000 data set. Taking into account the inferred distance of $9.2 \mathrm{kpc}$, the average blackbody radius during the decay of the burst was $\sim 6.7 \mathrm{~km}$. This value is within the range commonly observed (e.g. Van Paradijs 1978, also Cocchi et al. 2000 for recent results). For a canonical $1.4 M_{\odot}$ neutron star with $R=$ $10 \mathrm{~km}$, we derive an upper limit of $4.0 \times 10^{-10} M_{\odot} \mathrm{yr}^{-1}$ $\left(8.3 \times 10^{3} \mathrm{~g} \mathrm{~cm}^{-2} \mathrm{~s}^{-1}\right)$ to the accretion rate.

\subsection{A new class of low-luminosity bursters?}

SAX J1752.3-3138 is not the only example of a WFCdiscovered X-ray burster with no persistent emission observed. In 't Zand et al. (1998), already reported on the discovery of two burst sources (SAX J1753.5-2349, SAX J1806.5-2215) with no measured steady emission. Also GRS 1741.9-2853 and 1RXS J171824.2-402934 did not show persistent emission at the time when bursts were discovered (Cocchi et al. 1999b; Kaptein et al. 2000), even though GRS 1741.9-2853 was once detected with a luminosity of $\sim 2 \times 10^{36} \mathrm{erg} \mathrm{s}^{-1}$ in 1990 (Sunyaev 1990). The upper limits on their luminosities not being very constraining (of the order of $10^{36} \mathrm{erg} \mathrm{s}^{-1}$ ), it is unclear if these sources are to be regarded as weak persistent emitters instead of transients. But, as X-ray bursts are rarely observed (in $4.5 \mathrm{y}$ WFC monitoring, 3 from SAX J1806.5-2215 and GRS 1741.9-2853, only 1 from SAX J1752.3-3138, SAX J1753.5-2349 and 1RXS J171824.2-402934), the possibility that these sources are persistent emitters with luminosities around or just below $10^{36} \mathrm{erg} \mathrm{s}^{-1}$ seems unlikely. In fact more regular bursting behaviour is to be expected in this case, as typically observed in the medium-luminosity (atoll) NS LMXB (LvPT93).

So these sources could be usually quiescent systems where occasional mass transfer to the neutron star occurs, thus causing type I bursts. If so, they should not be very different from other weak transients observed by the WFCs, where burst activity was observed several days after the outburst (e.g. XTE J1709-267, SAX J1712.6-3739, SAX J1750.8-2900, SAX J1810.6-2609, SAX J1808.4-3658, see e.g. in 't Zand et al. 2000). The rather large distance (in excess of $8 \mathrm{kpc}$ for SAX J1752.3-3138 and GRS 1741.9-2853) and the incomplete time coverage by sensitive enough all-sky monitors could explain the missed detection of the (reasonably weak, a few $10^{36} \mathrm{erg} \mathrm{s}^{-1}$ ) outbursts triggering the occasional type I burst activity of these sources.

There is another intriguing possibility left: this small sample of burst-only objects could be part of a class of intrinsically weak persistent bursters $\left(L \lesssim 10^{35} \mathrm{erg} \mathrm{s}^{-1}\right)$, whose observation would be very useful to investigate the type I bursting behaviour at low accretion rates.
The sporadic burst activity of such sources would be then related to their unusual accretion regime instead of their transient behaviour. Actually this could be the case for 1RXS J171824.2-402934, whose ROSAT observations suggest weak persitent (even though variable) activity, with bolometric luminosity, taking into account the inferred distance of $\sim 7 \mathrm{kpc}$ (Kaptein et al. 2000), not exceeding $\sim 2 \times 10^{35} \mathrm{erg} \mathrm{s}^{-1}$. Moreover, an X-ray burst at very low persistent luminosity $\left(L \lesssim 10^{33} \mathrm{erg} \mathrm{s}^{-1}\right.$ ) was observed by Gotthelf \& Kulkarni (1997) in the globular cluster M 28. But that burst was notably subluminous $\left(L_{\text {peak }} \sim 4 \times 10^{36} \mathrm{erg} \mathrm{s}^{-1}\right)$, perhaps due to magnetic confinement of the burning fuel (Gotthelf \& Kulkarni 1997), while the bursts in the WFC sample reached peak luminosities of $\sim 10^{38} \mathrm{erg} \mathrm{s}^{-1}$, commonly observed in NSLMXBs. Further measurements with more sensitive instruments could unveil the nature (transient or weakly persistent) of these burst-only sources.

Acknowledgements. We thank the staff of the BeppoSAX Science Operation Centre and Science Data Centre for their help in carrying out and processing the WFC Galactic Centre observations. The BeppoSAX satellite is a joint Italian and Dutch program. M.C., A.B., L.N. and P.U. thank Agenzia Spaziale Nazionale $(A S I)$ for grant support. M.C. also thanks M. Federici (IAS staff) for technical support.

\section{References}

Boella, G., Butler, R. C., Perola, G. C., et al. 1997, A\&AS, 122,299

Bildsten, L. 2000, proc. 10th Ann. October Astrophys. Conf., ed. S. S. Holt, \& W. W. Zhang [astro-ph/0001135]

Cocchi, M., Bazzano, A., Natalucci, L., et al. 1999a, IAUC, 7307

Cocchi, M., Bazzano, A., Natalucci, L., et al. 1999b, A\&A, 346, L45

Cocchi, M., Bazzano, A., Natalucci, L., et al. 2000, Proc. 4th INTEGRAL Workshop [astro-ph/0105112]

Dickey, J. M., \& Lockman, F. J. 1990, A\&AR, 28, 215

Gotthelf, E. V., \& Kulkarni, S. R. 1997, ApJ, 490, L161

Heise, J. 1998, Nucl. Phys. B 69/1-3, 186

Heise, J., in 't Zand, J. J. M., Smith, M. J. S., et al. 1999, Astrophys. Lett. and Comm., 38/1-6, 297

Hoffman, J. A., Marshall, H. L., \& Lewin, W. H. G. 1978, Nature, 271, 630

Jager, R., Mels, W. A., Brinkman, A. C., et al. 1997, A\&A, 125,557

in 't Zand, J. J. M., Heise, J., Muller, J. M., et al. 1998, Nucl. Phys. B, 69/1-3, 228

in 't Zand, J. J. M. 2000, Proc. 4th INTEGRAL Workshop [astro-ph/0104299]

Kaptein, R. G., in 't Zand, J. J. M., Kuulkers, E., Heise, J., \& Cornelisse, R. 2000, A\&A, 358, L71

Lewin, W. H. G., van Paradijs, J., \& Taam, R. E. 1993, Space Sci. Rev., 62, 223

Sunyaev, R. A. 1990, IAUC, 5104

Ubertini, P., Bazzano, A., Cocchi, M., et al. 1999, Astrophys. Lett. and Comm., 38/1-6, 301

van Paradijs, J. 1978, Nature, 274, 650

van Paradijs, J., \& Mc Clintock, J. E. 1994, A\&A, 290, 133 